\title{
Knowledge, attitude, and practice of clinicians about antimicrobial stewardship and resistance among hospitals of Pakistan: a multicenter cross-sectional study
}

\author{
Sohaib Ashraf ${ }^{1}$ (D) Shoaib Ashraf ${ }^{2} \cdot$ Moneeb Ashraf $^{3} \cdot$ Muhammad Ahmad Imran $^{4} \cdot$ Zawar Ahmad Choudhary $^{5}$. \\ Hadiqa Tul Hafsa ${ }^{4}$. Abeer Bin Awais ${ }^{5} \cdot$ Larab Kalsoom $^{6}$. Iqra Farooq ${ }^{7}$. Zaighum Habib ${ }^{8}$. Sidra Ashraf ${ }^{9}$. \\ Qurrat UI Ain Iqbal ${ }^{5}$. Muhammad Ghufran ${ }^{10}$. Syed Sami Hussain Sherazi ${ }^{11}$. Muhammad Kiwan Akram ${ }^{12}$. \\ Rutaba Akmal ${ }^{13}$. Sundas Rafique ${ }^{14}$. Khawar Nawaz ${ }^{15}$. Zartasha Safdar ${ }^{11}$ • Uzma Nasim Siddique ${ }^{5}$. \\ Muhammad Hassan ${ }^{5}$. Shahroze Arshad ${ }^{5}$. Abdul Rehman Virk ${ }^{5}$ - Muhammad Ashraf ${ }^{11}$ • Qazi Abdul Saboor ${ }^{1}$. \\ Ayesha Humayun $^{16}$. Mateen Izhar ${ }^{4}$
}

Received: 13 April 2021 / Accepted: 23 August 2021 / Published online: 6 September 2021

(C) The Author(s), under exclusive licence to Springer-Verlag GmbH Germany, part of Springer Nature 2021

\begin{abstract}
Considering that antimicrobial resistance (AMR) is a global challenge, there is a dire need to assess the knowledge, attitude, and practice (KAP) of clinicians in AMR endemic countries. The current multicenter, cross-sectional study aimed at highlighting gaps in antimicrobial (AM) stewardship and AMR among practicing doctors working in public tertiary care teaching hospitals of Lahore, Pakistan. A KAP survey, based on a self-administered questionnaire containing 45 questions, was distributed among 336 clinicians in 6 randomly selected hospitals. Overall, $92 \%$ of the clinicians considered AMR as a worldwide problem but only $66 \%$ disagreed that cold and flu symptoms require antibiotics. Moreover, around $68 \%$ of the doctors felt confident about their practice in AM but still, $96 \%$ felt the need to get more knowledge about AM drugs. The need for refresher courses on rational antibiotic use was expressed by $84 \%$ of the participants. The main contributing factors considered for AMR by the doctors included excessive AM usage in the medical profession $(87.1 \%)$ and multiple antibiotics per prescription $(76.4 \%)$. Pharmacologically, AM spectrum was accurately chosen by $1.4 \%$ for Ampicillin, $0.003 \%$ for Erythromycin and $0 \%$ for Levofloxacin. Clinically, more than $50 \%$ of the clinicians used miscellaneous AM for empirical therapy of respiratory tract infection and cholecystitis. The data was analyzed using Statistical Package for Social Sciences (SPSS) version 25. It is concluded that the knowledge of clinicians is relatively poor for AM spectrum and drugs of choice for certain infections. However, the clinicians are aware of their shortcomings and desire for improvement.
\end{abstract}

Responsible Editor: Lotfi Aleya

Sohaib Ashraf, Shoaib Ashraf, Moneeb Ashraf and Muhammad Ahmad Imran joint first author

Zawar Ahmad Choudhary, Hadiqa tul Hafsa, Abeer-bin-Awais, Larab Kalsoom, Iqra Farooq joint second author

Sohaib Ashraf

sohaib-ashraf@outlook.com; sohaib@skzmdc.edu.pk; sashraf1@mgh.harvard.edu

Shoaib Ashraf

shoaib.ashraf@mail.mcgill.ca; sashraf@mgh.harvard.edu

Moneeb Ashraf

moneeb.ashraf@hotmail.com

Muhammad Ahmad Imran

ahmad.imran@skzmdc.edu.pk

Extended author information available on the last page of the article
Keywords Antimicrobials - Resistance - Antimicrobial use · Antibiotic misuse $\cdot$ Antibiotic resistance

\section{Introduction}

Although there were significant advances in antimicrobial therapy in the 1980s as the third-generation cephalosporin and new fluoroquinolones proved to be highly effective, the widespread usage of these newly developed drugs soon gave rise to a new problem. After a decade of antibiotic use, clinicians started to see persistent infections despite appropriate AM regimens. Newer strains of multi-drug resistant staphylococci, enterococci and streptococci were identified (Cosgrove 2006; Levy 1998; Okeke et al. 2005; World Health Organization 2012). With emerging antimicrobial resistance (AMR), there is a concordant rise in morbidity and mortality 
ratios (Hofer 2019). AMR-associated higher morbidity and mortality rates increase the economic burden on the health care sector especially in low-income countries (World Health Organization 2012). Factors responsible for AMR in third-world countries include over-prescription, unnecessary prescribing, incomplete treatment courses, self-medication, as well as insufficient infection control measures to prevent the spread of resistant bacteria in the community and hospitals (Okeke 2010; Sosa et al. 2010). The first step to deal with AMR is to educate the related staff about the overexpanding AMR dilemma (Dellit et al. 2007; Pulcini et al. 2007). This knowledge has led the USA, France, and Scotland to immediately develop a national recommendations' program to improve antibiotic stewardship in their countries (Dellit et al. 2007; Nathwani 2006; Pulcini et al. 2007). In certain studies, it is known that misuse of antibiotics by the general population increases the chance of getting infected with resistant strains of bacteria, yielding higher morbidity and mortality (Costelloe et al. 2010; Woodford and Livermore 2009). Meanwhile, the best approach to control the spread of AMR is to minimize antimicrobial usage and it can be achieved by changes in prescribing behaviors (García et al. 2011; Giblin et al. 2004; Guerra et al. 2007; Pulcini et al. 2011; Srinivasan et al. 2004; Wester et al. 2002). Factors thriving prescribing behavior of medical doctors can be analyzed by evidence-focused questionnaires such as knowledge, attitude, and practice (KAP) surveys.

In addition, the available studies have all been conducted in Europe, USA, China, Brazil, and Peru but few studies were done in Pakistan (Ahmed et al. 2020; Faizullah et al. 2017; García et al. 2011; Giblin et al. 2004; Shahid et al. 2017; Wester et al. 2002). Therefore, we conducted a KAP survey about antimicrobial usage and antimicrobial resistance among public tertiary care teaching hospitals in Lahore, Pakistan.

\section{Materials and methods}

\section{Study design}

We conducted a cross-sectional study in which a selfadministered questionnaire was distributed among physicians at the postgraduate trainee level and faculty in six randomly selected tertiary care teaching hospitals of Lahore, Pakistan. The variables were knowledge, attitude, and practice of doctors regarding antimicrobial usage and resistance. The inclusion criteria included clinicians working in medicine, surgery, and their allied departments. Psychiatrists, radiologists, and basic sciences doctors were excluded. The questionnaire was distributed on-site during working hours in hard copy along with the option of filling it electronically.

\section{Questionnaire design and grading method}

The questionnaire was developed in consultation with a group of experts on questionnaire design and infectious diseases, and after searching the literature for comparative studies (Guerra et al. 2007; Srinivasan et al. 2004; Wester et al. 2002). Before we finalized the questionnaire, it was reviewed by a panel of clinical experts of Shaikh Zayed Post-Graduate Medical Complex, Lahore, Pakistan. The questionnaire inquired the participants' awareness regarding the recent scope of antimicrobial resistance (15 questions), the clinical attitude contributing to the awareness ( 5 questions), factors influencing confidence regarding usage and prescription of AM and AMR (6 questions), knowledge regarding contributing factors of AMR (7 questions) and basic knowledge about the clinical indications, spectrum, administration, and pharmacology of antimicrobials (12 questions). The questions used a 4 or 5-point Likert scale and included answers ranging from "strongly agree" to "strongly disagree", from "very confident" to "not confident" and from "always" to "never". The participants were also asked to provide the correct AM spectrum of Ampicillin (Rafailidis et al. 2007), Metronidazole (Freeman et al. 1997) Levofloxacin (Anderson and Perry 2008; Zhanel et al. 2006), Erythromycin (Zhanel et al. 2001) Vancomycin, (Marsot et al. 2012) and Ceftriaxone (Lamb et al. 2002). The anonymous nature of the questionnaire and prior consent from all clinicians addressed ethical issues. This research was in accordance with principles 3 and 4 of the World Medical Association's Declaration of Helsinki. The questionnaire was submitted in a pilot test to 34 participants to check comprehension and clarity of the questions.

\section{Data collection and analysis}

Randomized selection of tertiary care teaching hospitals of Lahore by using lottery method was done which made us select Ganga Ram Hospital, Jinnah Hospital, Services Hospital, Mayo Hospital, Sheikh Zayed Hospital, and General Hospital. The sample size was calculated using www.epitool.ausvet.com with an estimated true proportion of 0.5 with a confidence level of $95 \%$ and desired precision of 0.05 , the population size was calculated to be 2614 doctors. The doctors were contacted after consultation with administrators/medical superintendents of respective hospitals, and the calculated sample size was 336. It was predefined that a questionnaire was to be considered valid for analysis if $90 \%$ of the questions were answered. The data was analyzed using IBS SPSS software version 25 (SPSS Inc., Chicago, IL, USA) 


\section{Results}

\section{Demographics}

All participants had at least a Bachelor of Medicine and Bachelor of Surgery (MBBS) degree with a mean age of 31 years. Of the total, 336 filled questionnaires, $84.5 \%$ (284) met the selection criteria. Among those, $34.5 \%$ were males and $25.7 \%$ were faculty members (Table 1 ).

\section{Knowledge about antibiotics and AMR}

Knowledge about the empiric therapy of various common infections was inquired (Fig. 1A). For urinary tract infection (uncomplicated), around one-half of the doctors prescribed ciprofloxacin, while Levofloxacin and Trimethoprimsulfamethoxazole (TMP-SMZ) were written by $11.4 \%$ and $5.6 \%$, respectively, as a first-line therapy. Upper respiratory tract infection (uncomplicated) was being managed through supportive therapy as per the World Health Organization (WHO) recommendations by only $3.9 \%$ of clinicians while one-fourth were managing it with amoxicillin. Levofloxacin and azithromycin were being prescribed by $5.6 \%$ and $5.9 \%$ of the clinicians, respectively. Shockingly, more than $70 \%$ were prescribing miscellaneous AM drugs. In clinical practice, doctors considered ciprofloxacin and Metronidazole in around

Table 1 Demographic characteristics of participants

\begin{tabular}{lll}
\hline & & Number (percentage) \\
\hline Gender & Male & $98(34.5)$ \\
Years of experience & Female & $186(65.5)$ \\
& $\leq 5$ & $198(69.72)$ \\
Age & $5-10$ & $37(13.03)$ \\
Hospital & $\geq 10$ & $49(17.25)$ \\
& $\geq 40$ & $47(16.55)$ \\
& $<40$ & $237(83.45)$ \\
Position & SZH & $58(20.4)$ \\
& JHL & $57(20.1)$ \\
& Mayo & $60(21.1)$ \\
& General & $55(19.4)$ \\
& SIMS & $54(19.0)$ \\
& Post Graduate Trainees & $211(74.3)$ \\
& Medical Officer & $30(10.6)$ \\
& Assistant Professor & $18(6.3)$ \\
Department & Associate Professor & $11(3.9)$ \\
& Professor & $14(4.9)$ \\
& Medicine & $82(28.9)$ \\
& Medicine Allied & $93(32.7)$ \\
& Surgery & $47(16.5)$ \\
& Surgery Allied & $62(21.8)$ \\
\hline
\end{tabular}

$31 \%$ and $40 \%$ of their prescriptions, respectively, as the first-line treatment of gastroenteritis while only $11 \%$ decided to manage it conservatively. The first-line treatment for community-acquired pneumonia chosen by approximately $13 \%$ of doctors was amoxicillin, Levofloxacin, and Ceftriaxone individually, and azithromycin was prescribed by $7 \%$ of them. Whereas more than $50 \%$ of prescriptions were contributed by miscellaneous drugs. Although most of the clinicians $(56.1 \%)$ prescribed penicillin as the first-line choice for the treatment of cellulitis, while others chose miscellaneous drugs $(26.9 \%)$ along with amoxicillin $(9.6 \%)$ and Ceftriaxone $(7.4 \%)$. As far as cholecystitis was concerned, the majority disputed on the first line antimicrobial by choosing miscellaneous drugs (57.2\%).

Knowledge about the antimicrobial spectrum was also evaluated (Fig. 1B). $1.4 \%$ of the clinicians chose the correct spectrum of Ampicillin. However, $88.3 \%$ were inclined towards gram-positive cocci, $39.2 \%$ considered it to be active against gram-positive bacilli, $31.8 \%$ believed it to be active against gram-negative cocci, $14.3 \%$ suggested it as a preferred treatment for gram-negative bacilli and $2.6 \%, 10 \%$, and 3.3\% marked anaerobes, spirochetes, and atypical bacteria respectively. Amazingly, the correct antimicrobial spectrum of Metronidazole was selected by $61.6 \%$. However, $85 \%$ of doctors do know that it is effective against anaerobes while $13.3 \%$ considered its coverage for gram-positive cocci and other lesser percentages of doctors considered it to be effective against other organisms. Astonishingly, none of the clinicians were able to identify the correct antimicrobial spectrum of Levofloxacin. On the positive side, greater than half of the doctors knew its effectiveness against gram-positive cocci, more than $1 / 3$ considered it effective against gram-positive and gram-negative bacilli whereas $20 \%$ correctly identified it to be effective against atypical bacteria. However, around $40 \%$ of the doctor community also thought it to be effective against gram-negative cocci. Only one doctor $(0.003 \%)$ came up with the correct antimicrobial spectrum for Erythromycin but $68.2 \%$ of the doctors considered its coverage against gram-positive cocci to be true. Between 25 and $35 \%$ of the doctors contemplated its effectiveness against, gram-negative bacilli, gram-negative cocci, gram-positive bacilli, and atypical bacteria. Around 5.9\% considered it to be effective against spirochetes but $6.8 \%$ believed that it also acted on anaerobes. Interestingly, $0.4 \%$ picked the correct spectrum of Vancomycin, 77.4\% chose gram-positive cocci while gramnegative cocci and gram-positive bacilli were chosen by around $33 \%$ of clinicians. Anaerobes, spirochetes, and atypical were marked by less than $10 \%$ of the doctors. At least $2.8 \%$ of the doctors described the correct spectrum of Ceftriaxone. $53.9 \%$ selected gram-positive cocci as the right option whereas $66.3 \%$ of gram-negative cocci. Gram-positive bacilli were chosen as the correct option by $29.1 \%$ while $39.1 \%$ described gram-negative bacilli as the correct option. 
A Knowledge about the empirical treatment of various infections.
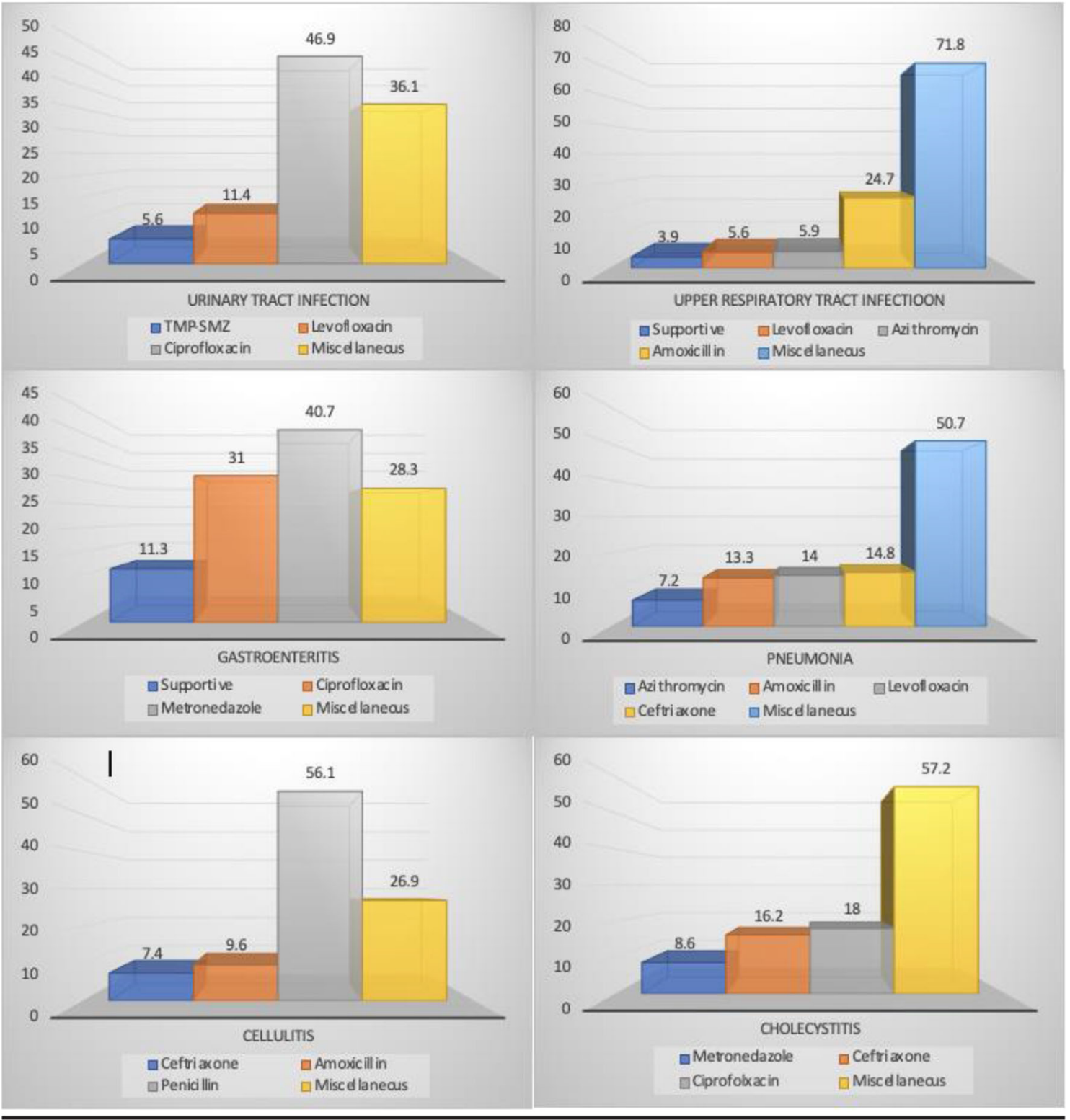

Fig. 1 A Knowledge about the empirical treatment of various infections. B Knowledge about the antimicrobial spectrum of the drugs

Anaerobes (9.4\%), spirochetes (11.3\%), and atypical (10.7\%) were marked.

\section{Attitudes regarding antibiotics and AMR}

In our study (Fig. 2A), 92\% of the clinicians considered AMR as a worldwide problem but only $66 \%$ disagreed that cold and flu-like symptoms require antibiotics. And even though $68.4 \%$ of doctors felt confident about their knowledge and practice in the antibiotic prescription, $96 \%$ felt necessary to get more knowledge about AM. Along with that, $20 \%$ believed AM prescription would not cause damage. Furthermore, $78 \%$ of the doctors felt that poor manufacturing practices of antibiotics had made them ineffective, while $35 \%$ believed efficacy would be better with newer costly antibiotics. Moreover, $60 \%$ of the physicians believe that patient's demand has led to over-prescribing while $17 \%$ considered over-prescription better than under-prescription in infected patients and 37\% agreed that antimicrobials should be available over the counter. In addition to this, $19.4 \%$ believed AMR is not a problem in their clinical settings whereas $84 \%$ expressed the need for refresher courses on rational antibiotic use and to carry out large scale campaigns for antibiotic awareness (39\%).

The factors which were found to be the top causes of antibiotic resistance in our study included: multiple 
B Knowledge about the antimicrobial spectrum of the drugs.
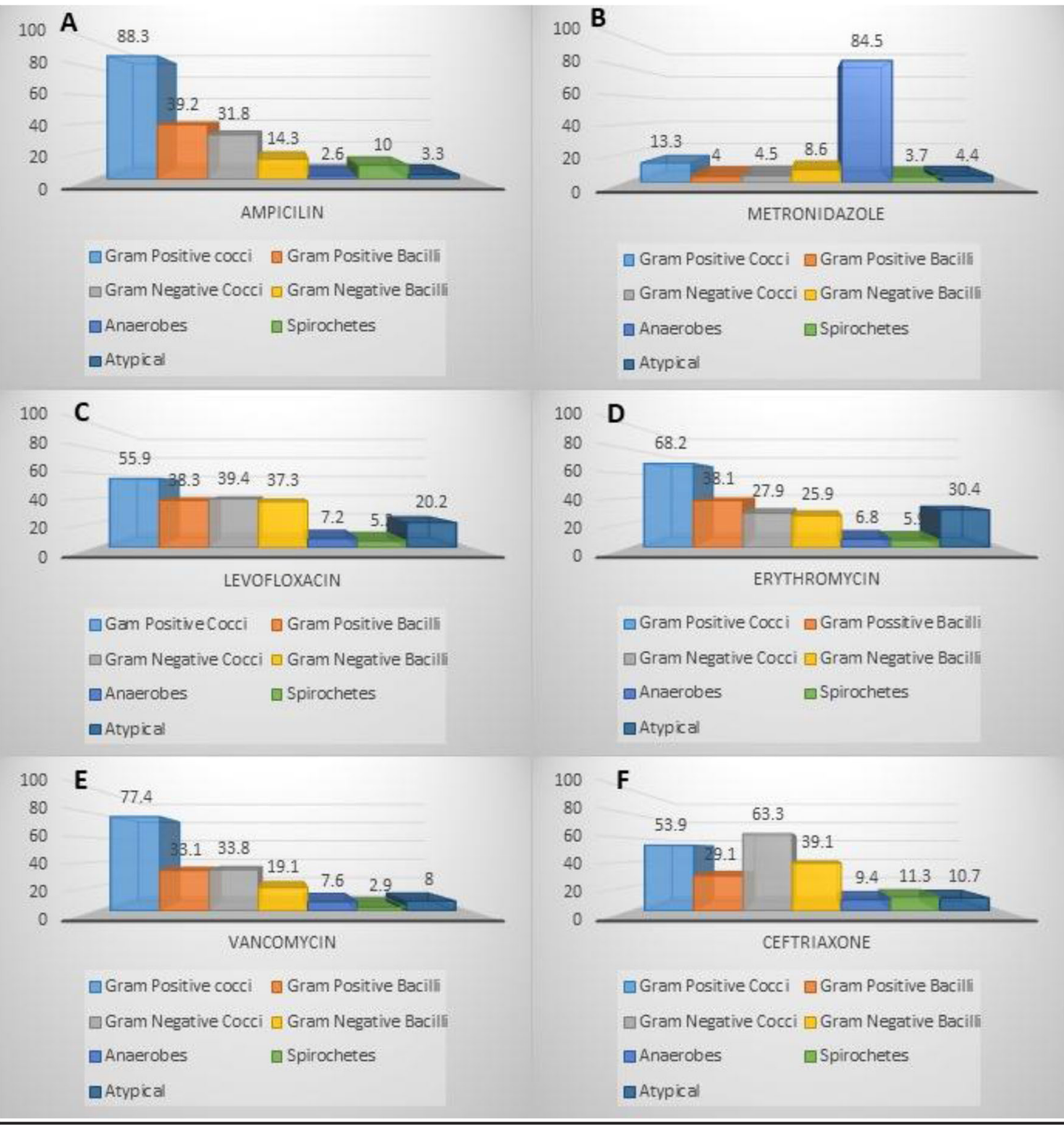

Fig. 1 continued.

antibiotics per prescription (76.4\%) and excessive use of AM in daily life (87.1\%). The factors most frequently identified as less important included: prescribing too many broad-spectrum antibiotics $(61.7 \%)$, prescribing low dose antibiotics (60.5\%), longer duration of AM usage (66.2\%), and poor hand hygiene (54.9\%). The factor which the study found to be least important was paying too much attention to advertising (36.3\%) (Fig. 2B).

\section{Practices regarding antibiotics and AMR}

According to the study (Fig. 3A), antibiotics were prescribed confidently by $75.4 \%$ of doctors and $81 \%$ of them felt confident while planning the duration of AM treatment whereas $74.3 \%$ felt confident while stopping treatment. More than $75 \%$ of the respondents felt confident in selecting the correct dose and duration of administration, and in using combination therapy. A total of $82.4 \%$ of doctors were also confident that they could choose between intravenous and oral routes correctly.

While $65 \%$ of doctors felt most confident about prescribing antibiotics, only $40 \%$ considered sometimes AMR while prescribing. Half of the respondents agreed that doctors sometimes get influenced by marketing strategies of multinational companies while approximately $1 / 4$ of the participants believed that it occurred more often. 
A Attitudes Regarding Antibiotics and AMRamong 284 participants (data in the graph represents percentages)

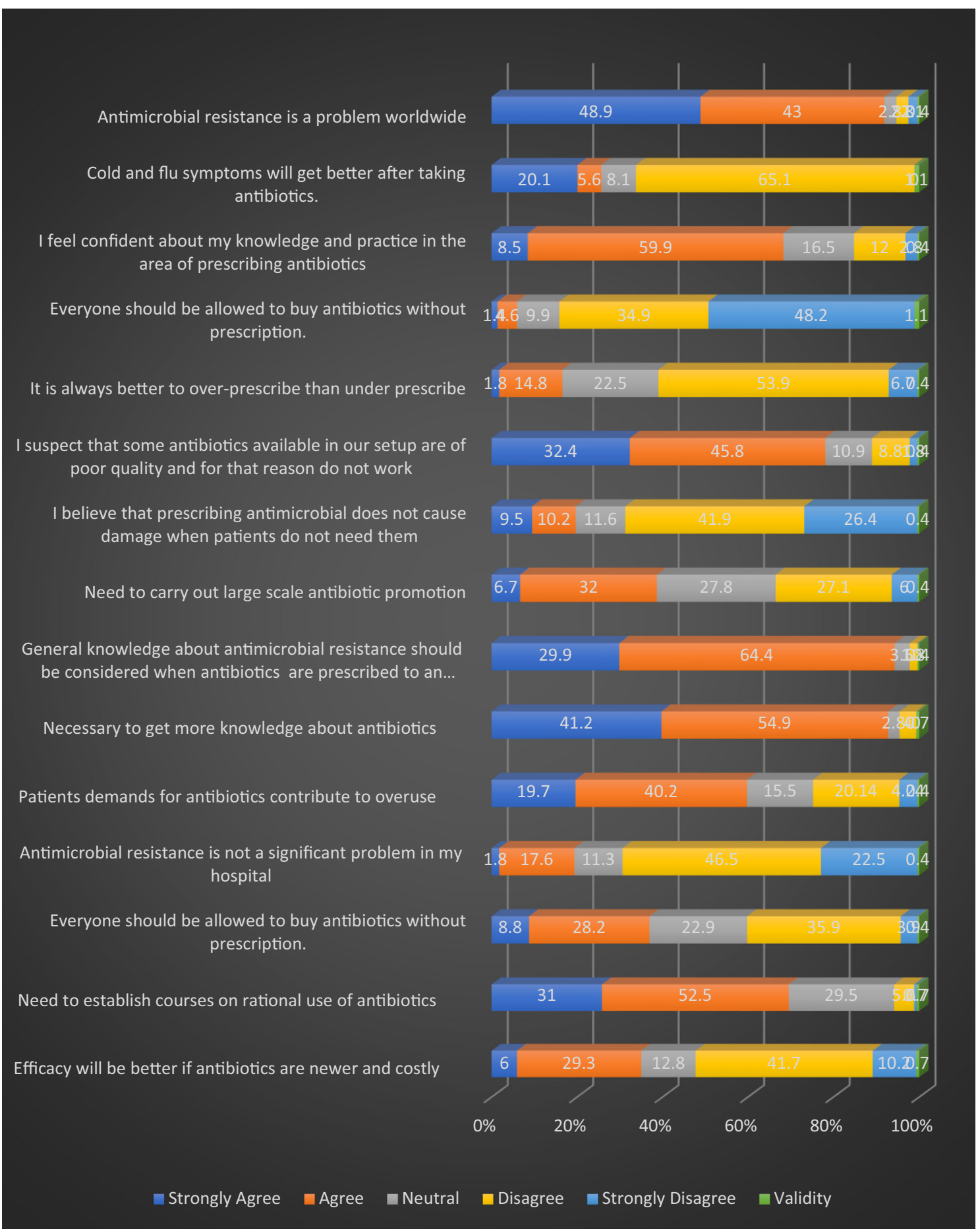

Fig. 2 A Attitudes Regarding Antibiotics and AMR among 284 participants (data in the graph represents percentages). B Factors influencing the clinicians' practice leading to AMR. Data represent percentages among 284 participants

Interestingly, $50 \%$ (sometimes) and $30 \%$ (mostly) reviewed their choice for antibiotic usage with their senior colleagues. However, among doctors $71 \%$ (sometimes) and $11 \%$ (mostly) were guided with a different antibiotic (Fig. 3B).

\section{Discussion}

This survey demonstrated the knowledge, attitude, and practices of doctors regarding AMR in six large public teaching hospitals in Lahore. From the study, it was evident that the 
B Factors influencing the clinicians' practice leading to AMR. Data represent percentages among 284 participants

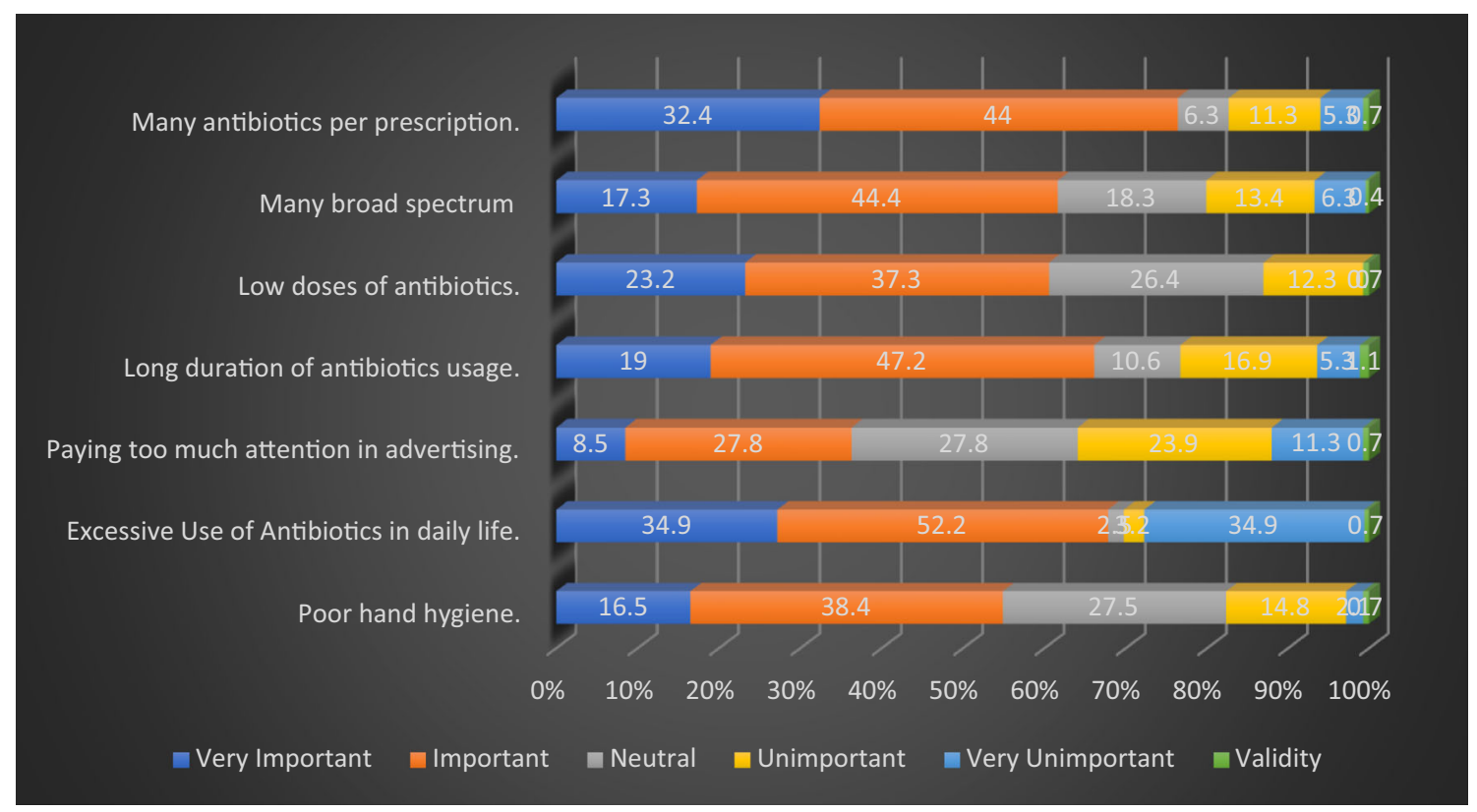

Fig. 2 continued.

knowledge about AMs, their misuse, and the concern regarding the emerging resistance problem worldwide was evident. However, quite astonishingly, most of the physicians were using AMs even for mild upper respiratory tract infections. Moreover, their attitude for prescribing multiple antibiotics per prescription was also debatable. Most of the doctors' knowledge regarding AM spectrum and empirical treatment for certain infections was not up to date. This shows that special attention should be given to continuous medical education, and appropriate interventions must be used to improve the knowledge of doctors in this area. It was evident from the study that $91.9 \%$ of participants considered AMR to be a rising concern, yet AMR was completely ignored in their practice. A study conducted in Ghanaian tertiary care hospital also assessed similar parameters of physicians. $30.1 \%$ and $42.9 \%$ of the physicians graded the level of worldwide growing antibiotic resistance as very important and important respectively (Labi et al. 2018). The majority agreed on the fact that the main reason for such growing resistance is the daily irrational use of AMs. Similar results were seen in a study conducted in New York where 32\% of respondents "usually" or "always" preferred using the antibiotic with broadest spectrum in their empiric therapies while $29 \%$ of participants did "sometimes" did it (Salsgiver et al. 2018). A small ray of hope was observed as the young doctors were witnessed seeking help from experienced colleagues when prescribing, irrespective of their specialization (department) so that the appropriate antibiotic could be given to avoid resistance. However, $40 \%$ of participants responded that they did not consult their peers. $19.4 \%$ of the participants shed light on the issue that overuse of AMs is a consequence of patient's demand, which is due to their limited understanding of appropriate antibiotic use. A stark contrast was seen in Italy where a national crosssectional study was done to evaluate the knowledge and behavior of young Italian doctors. Over there, $68 \%$ of doctors stated that they "never" prescribe antibiotics when the patient expects or asks for it (Di Gennaro et al. 2020). They also need to know that these drugs do not affect viral infections. These results in comparison to the study done in a hospital in Peru that showed patient's pressure to prescribing antimicrobials was considered as a major contributing factor $(72 \%)$ to AM overuse in the community (García et al. 2011). Similarly, a study from Alexandria university teaching hospital also concluded that antibiotic prescribing trends of physicians were influenced by patient's demands (78.5\%) and socioeconomic status (76.3\%) (El-Hawy et al. 2017). We have concluded that awareness of the public through community-targeted media is immensely important. More than half of the participants agreed that AMs in their hospitals are of poor quality, although we have not explored in detail the definition of "poor" quality. The two main reasons for such substandard quality of medicines include the counterfeit drugs detected in Lahore. Secondly, the use of generic drugs is frequently perceived to be less effective. This affects the physician's ability to treat a disease effectively as well as the doctor-patient relationship. The Pakistan Ministry of Health should take some serious action as it is an important issue that directly relates to patient health. The availability of standardized antibiotics should be made possible so that the treatment would not be compromised. 
A Clinicians' practice regarding antibiotics. Data represent percentages among 284 participants

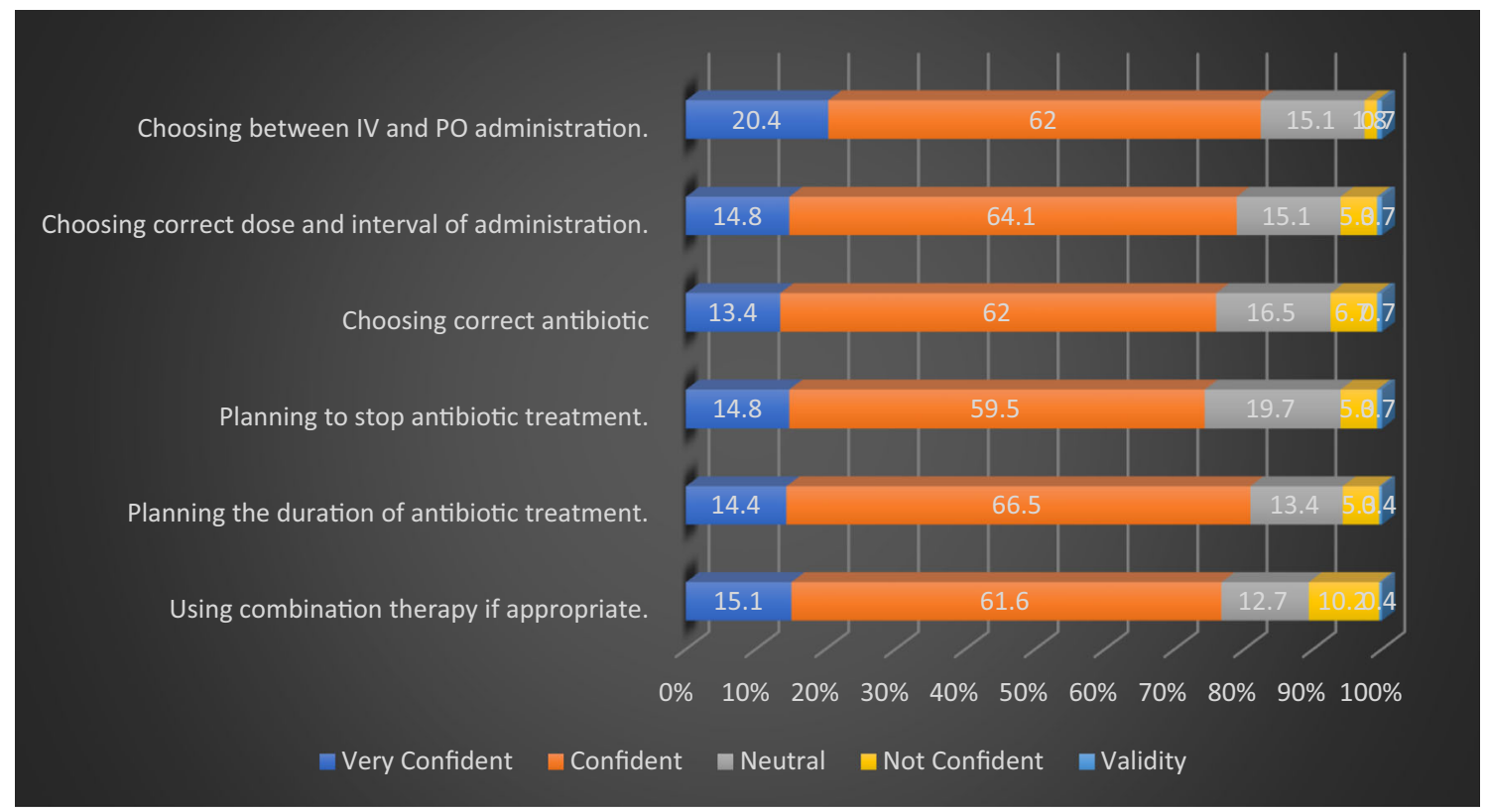

B Clinical practices leading to AMR. Data represent percentages among 284 participants.

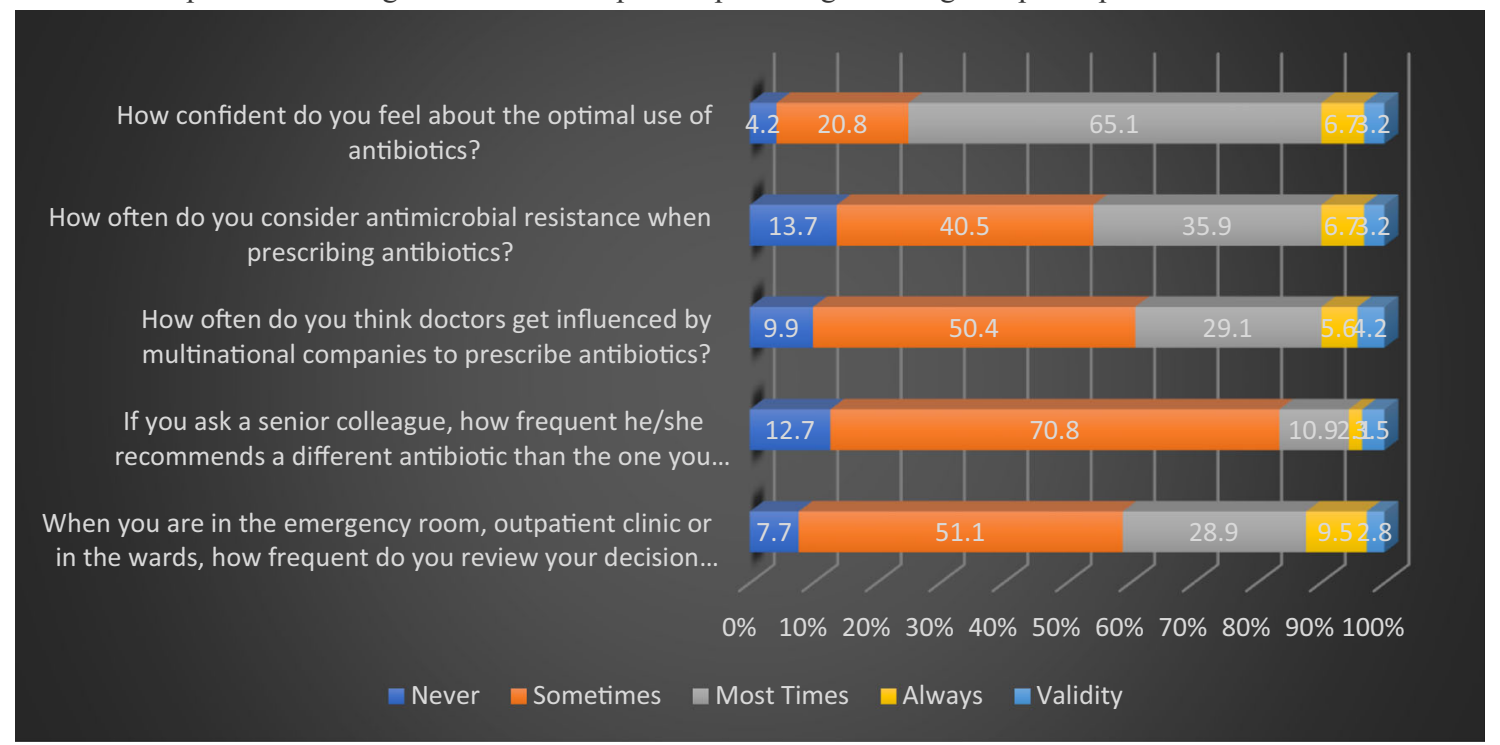

Fig. 3 A Clinicians' practice regarding antibiotics. Data represent percentages among 284 participants. B Clinical practices leading to AMR. Data represent percentages among 284 participants

To the best of our knowledge, this is the first multicenter cross-sectional study assessing knowledge, attitude, and practice of antimicrobials and antimicrobial resistance of clinicians in Lahore, Pakistan. A uni-center study conducted in Services Institute of Medical Sciences, Pakistan, also concluded that antibiotic overuse has become a major problem as $70 \%$ of physicians were prescribing more than one antibiotic per day and $80 \%$ of the physicians reviewed their decision to prescribe antibiotics by discussing with an experienced colleague. It showed that the knowledge of freshly graduated physicians regarding antibiotic prescription was not up to the mark (Shahid et al. 2017). Similarly, a study conducted among healthcare workers in Iran showed that $88 \%$ agreed on the establishment of local guidelines and $94 \%$ believed that education regarding antibiotics can help reduce AMR (Firouzabadi and Mahmoudi 2020). Our study findings are coherent with the previously done KAP surveys (Ahmed et al. 2020; Di Gennaro et al. 2020; Faizullah et al. 2017; Firouzabadi and Mahmoudi 2020; García et al. 2011; Giblin et al. 2004; Labi et al. 2018; Salsgiver et al. 2018; Shahid et al. 2017; Wester et al. 2002). 
Educational and awareness programs regarding the vigilant use of antimicrobials and AMR should be conducted on a regular basis. Mandatory continuing medical education courses should be attended by the healthcare professionals to explain their role in limiting the misuse of AMs and emerging AMR. Moreover, active educational techniques including clinical scenarios that simulate real clinical settings, objective-centered clinical examinations, awareness campaigns, and interactive learning workshops should be regularly conducted (Yang et al. 2016). Future studies from distant, primary, and secondary health centers are recommended.

The anonymous nature of the questionnaire and voluntary involvement provided reliable answers with no social pressure. Despite the strengths, the study also has some potential limitations. Firstly, most of the participants (74.3\%) were postgraduate trainees in their respective departments. Additionally, a stringent criterion was used to access the knowledge of AM spectrum which may not be clinically relevant. Intrinsic limitations of this cross-sectional study design include the assessment of exposure and outcome are simultaneous without evidence of any temporal relationship between the two. Hence, without longitudinal data, it is impossible to establish a true cause and effect relationship. Another limitation of the study was that the participants from non-teaching hospitals and rural/basic health centers were not interviewed. Moreover, in a KAP survey, sometimes the physicians get socially pressured and give more socially acceptable answers rather than expressing their true opinions. Therefore, to reduce this potential bias, incognito participation was made certain and the case-based questions about AM prescription were composed. Another problem was that consultants working in hospitals did not give due attention to the lengthy questionnaire so it would be appropriate to suggest that further studies should be done to access the knowledge, attitudes, and practice about AM use among physicians from community centers. Lastly, self-selection bias cannot be neglected considering volunteer participations of doctors in the study as their motivation for study enrollment significantly stood them out of the target population. As this study was conducted in six large, public, tertiary-level teaching hospitals, and a significant number of qualified prescribing doctors were a part of this, we are positive that the outcomes will apply to other public hospitals in Pakistan. Future studies from peripheral health centers are recommended.

\section{Conclusion}

The present KAP survey has generated information about the knowledge, prescribing attitudes, and practices of medical doctors among all public tertiary care teaching hospitals of Lahore, Pakistan. It highlighted the areas that need improvement to prevent AMR. The knowledge of clinicians is relatively poor when it comes to drug spectrums and drugs of choice for certain infections. However, the clinicians know about their shortcomings, and they have a positive attitude towards professional development.

Acknowledgements The authors would like to thank the respondents who participated in this survey. We would like to thank Prof. Ali Ahmad, Department of Microbiology, Infectiology and Immunology, Centre Hospitalier Universitaire (CHU) Sainte Justin/University of Montreal, Montreal, Canada, for his intellectual input and proofreading this article. We would also want to pay our fellow colleague Prof. Dr. Zai Ullah who lost his life in battle against COVID-19.

Author contribution SoA, ShA, MoA, and MAI drafted the first draft and all authors participated in writing subsequent drafts. SoA, MoA, MAI, and $\mathrm{ZA}, \mathrm{AbA}$, contributed to collecting data and its interpretation. ZA, SoA, MAI, and MKA drafted the figures and the Table. AH, QaS, MA, and MI supervised and edited the follow-up drafts. All authors approved the final manuscript.

Funding Smile Welfare Organization.

Data availability The data used during the current study are available from the corresponding authors on reasonable request.

\section{Declarations}

Ethics approval and consent to participate Ethical approval was taken from the institutional review board of Shaikh Zayed Post-Graduate Medical Institute, Lahore. Written informed consent was taken from participants for data collection and maintaining confidentiality.

\section{Consent for publication Not applicable.}

Competing interests The authors declare no competing interest and all the authors have seen and agreed to the submission of this paper.

\section{References}

Ahmed H, Bhimani S, Khanum I, Khan A, Khetpal A, Abbas MA, .. . Makhdoom IUHM (2020). Knowledge, attitude and perception survey of doctors regarding antibiotic use and resistance in Karachi, Pakistan. JPMA. The Journal of the Pakistan Medical Association, 70(6), 1023.

Anderson VR, Perry CM (2008) Levofloxacin. Drugs 68(4):535-565

Cosgrove SE (2006). The relationship between antimicrobial resistance and patient outcomes: mortality, length of hospital stay, and health care costs. Clinical Infectious Diseases, 42(Supplement_2), S82S89.

Costelloe C, Metcalfe C, Lovering A, Mant D, Hay AD (2010) Effect of antibiotic prescribing in primary care on antimicrobial resistance in individual patients: systematic review and meta-analysis. Bmj 340: c2096

Dellit TH, Owens RC, McGowan JE, Gerding DN, Weinstein RA, Burke JP et al (2007) Infectious Diseases Society of America and the Society for Healthcare Epidemiology of America guidelines for developing an institutional program to enhance antimicrobial stewardship. Clinical Infectious Diseases 44(2):159-177 
Di Gennaro F, Marotta C, Amicone M, Bavaro DF, Bernaudo F, Frisicale EM et al (2020) Italian young doctors' knowledge, attitudes and practices on antibiotic use and resistance: A national crosssectional survey. J Glob Antimicrob Resist 23:167-173. https:// doi.org/10.1016/j.jgar.2020.08.022

El-Hawy RM, Ashmawy MI, Kamal MM, Khamis HA, Abo El-Hamed NM, Eladely GI et al (2017) Studying the knowledge, attitude and practice of antibiotic misuse among Alexandria population. Eur J Hosp Pharm 24(6):349-354. https://doi.org/10.1136/ejhpharm2016-001032

Faizullah M, Nisar-ur-Rahman MIU, Anwar M, Sarfraz M (2017) A cross-sectional study on knowledge, attitude and practices of medical doctors towards antibiotic prescribing patterns and resistance in Khyber Pakhtun Khawah, Pakistan. J Appl Pharm Sci 7(12):38-46

Firouzabadi D, Mahmoudi L (2020) Knowledge, attitude, and practice of health care workers towards antibiotic resistance and antimicrobial stewardship programmes: a cross-sectional study. J Eval Clin Pract 26(1):190-196. https://doi.org/10.1111/jep.13177

Freeman CD, Klutman NE, Lamp KC (1997) Metronidazole. Drugs 54(5):679-708

García C, Llamocca LP, García K, Jiménez A, Samalvides F, Gotuzzo E, Jacobs J (2011) Knowledge, attitudes and practice survey about antimicrobial resistance and prescribing among physicians in a hospital setting in Lima, Peru. BMC Clin Pharmacol 11:18. https://doi. org/10.1186/1472-6904-11-18

Giblin TB, Sinkowitz-Cochran RL, Harris PL, Jacobs S, Liberatore K, Palfreyman MA et al (2004) Clinicians' perceptions of the problem of antimicrobial resistance in health care facilities. Archives of Internal Medicine 164(15):1662-1668

Guerra CM, Pereira C, Neves Neto A, Cardo DM, Correa L (2007) Physicians' perceptions, beliefs, attitudes, and knowledge concerning antimicrobial resistance in a Brazilian teaching hospital. Infect Control Hosp Epidemiol 28(12):1411-1414

Hofer U (2019) The cost of antimicrobial resistance. Nat Rev Microbiol 17(1):3. https://doi.org/10.1038/s41579-018-0125-x

Labi AK, Obeng-Nkrumah N, Bjerrum S, Aryee NAA, Ofori-Adjei YA, Yawson AE, Newman MJ (2018) Physicians' knowledge, attitudes, and perceptions concerning antibiotic resistance: a survey in a Ghanaian tertiary care hospital. BMC Health Serv Res 18(1):126. https://doi.org/10.1186/s12913-018-2899-y

Lamb HM, Ormrod D, Scott LJ, Figgitt DP (2002) Ceftriaxone. Drugs 62(7):1041-1089

Levy SB (1998) The challenge of antibiotic resistance. Scientific American 278(3):46-53

Marsot A, Boulamery A, Bruguerolle B, Simon N (2012) Vancomycin. Clinical pharmacokinetics 51(1):1-13

Nathwani D (2006) Antimicrobial prescribing policy and practice in Scotland: recommendations for good antimicrobial practice in acute hospitals. Journal of Antimicrobial Chemotherapy 57(6):11891196

Okeke IN (2010). Poverty and root causes of resistance in developing countries. In Antimicrobial resistance in developing countries (pp. 27-35): Springer
Okeke IN, Laxminarayan R, Bhutta ZA, Duse AG, Jenkins P, O'Brien TF, Pablos-Mendez A, Klugman KP (2005) Antimicrobial resistance in developing countries. Part I: recent trends and current status. The Lancet infectious diseases 5(8):481-493

Organization, W. H. (2012). The evolving threat of antimicrobial resistance: options for action: World Health Organization.

Pulcini C, Cua E, Lieutier F, Landraud L, Dellamonica P, Roger P (2007) Antibiotic misuse: a prospective clinical audit in a French university hospital. European journal of clinical microbiology \& infectious diseases 26(4):277-280

Pulcini C, Williams F, Molinari N, Davey P, Nathwani D (2011) Junior doctors' knowledge and perceptions of antibiotic resistance and prescribing: a survey in France and Scotland. Clinical microbiology and infection 17(1):80-87

Rafailidis PI, Ioannidou EN, Falagas ME (2007) Ampicillin/sulbactam. Drugs 67(13):1829-1849

Salsgiver E, Bernstein D, Simon MS, Eiras DP, Greendyke W, Kubin CJ, Mehta M, Nelson B, Loo A, Ramos LG, Jia H, Saiman L, Furuya EY, Calfee DP (2018) Knowledge, attitudes, and practices regarding antimicrobial use and stewardship among prescribers at acute-care hospitals. Infect Control Hosp Epidemiol 39(3):316-322. https:// doi.org/10.1017/ice.2017.317

Shahid A, Iftikhar F, Arshad MK, Javed Z, Sufyan M, Ghuman RS, Tarar Z (2017) Knowledge and attitude of physicians about antimicrobial resistance and their prescribing practices in Services hospital, Lahore, Pakistan. J Pak Med Assoc 67(6):968

Sosa ADJ, Byarugaba DK, Amábile-Cuevas CF, HsuehPR, Kariuki S, Okeke IN (2010). Antimicrobial resistance in developing countries: Springer

Srinivasan A, Song X, Richards A, Sinkowitz-Cochran R, Cardo D, Rand C (2004) A survey of knowledge, attitudes, and beliefs of house staff physicians from various specialties concerning antimicrobial use and resistance. Archives of Internal Medicine 164(13):1451-1456

Wester CW, Durairaj L, Evans AT, Schwartz DN, Husain S, Martinez E (2002) Antibiotic resistance: a survey of physician perceptions. Archives of Internal Medicine 162(19):2210-2216

Woodford N, Livermore DM (2009) Infections caused by Gram-positive bacteria: a review of the global challenge. Journal of Infection 59: S4-S16

Yang K, Wu D, Tan F, Shi S, Guo X, Min Q, Zhang X, Cheng H (2016) Attitudes and perceptions regarding antimicrobial use and resistance among medical students in Central China. Springerplus 5(1):1779. https://doi.org/10.1186/s40064-016-3454-0

Zhanel GG, Dueck M, Hoban DJ, Vercaigne LM, Embil JM, Gin AS, Karlowsky JA (2001) Review of macrolides and ketolides. Drugs 61(4):443-498

Zhanel GG, Fontaine S, Adam H, Schurek K, Mayer M, Noreddin AM, Gin AS, Rubinstein E, Hoban DJ (2006) A review of new fluoroquinolones. Treatments in respiratory medicine 5(6):437-465

Publisher's note Springer Nature remains neutral with regard to jurisdictional claims in published maps and institutional affiliations. 


\section{Affiliations}

\section{Sohaib Ashraf ${ }^{1}$ (D) - Shoaib Ashraf ${ }^{2} \cdot$ Moneeb Ashraf $^{3} \cdot$ Muhammad Ahmad Imran $^{4} \cdot$ Zawar Ahmad Choudhary $^{5}$. Hadiqa Tul Hafsa ${ }^{4}$ - Abeer Bin Awais ${ }^{5} \cdot$ Larab Kalsoom $^{6}$. Iqra Farooq ${ }^{7}$ - Zaighum Habib ${ }^{8} \cdot$ Sidra Ashraf $^{9}$. Qurrat UI Ain Iqbal ${ }^{5}$. Muhammad Ghufran ${ }^{10}$. Syed Sami Hussain Sherazi ${ }^{11}$. Muhammad Kiwan Akram ${ }^{12}$. Rutaba Akmal ${ }^{13}$. Sundas Rafique ${ }^{14}$. Khawar Nawaz ${ }^{15}$. Zartasha Safdar ${ }^{11}$. Uzma Nasim Siddique ${ }^{5}$. Muhammad Hassan $^{5}$. Shahroze Arshad ${ }^{5}$. Abdul Rehman Virk ${ }^{5}$ - Muhammad Ashraf ${ }^{11}$ • Qazi Abdul Saboor ${ }^{1}$. Ayesha Humayun $^{16}$. Mateen Izhar ${ }^{4}$}

1 Department of Cardiology, Shaikh Zayed Post-Graduate Medical Complex, Lahore, Pakistan

2 Department of Pathobiology, Riphah International University, Lahore, Pakistan

3 Department of Pharmacology, King Edward Medical University, Mayo Hospital, Lahore, Pakistan

4 Department of Microbiology, Shaikh Zayed Post-Graduate Medical Complex, Lahore, Pakistan

5 Department of Internal Medicine, Shaikh Zayed Post-Graduate Medical Complex, Lahore, Pakistan

6 Department of Internal Medicine, Services Institute of Medical Sciences, Lahore, Pakistan

7 Department of Pediatrics Surgery, Children Hospital and Institute of Child Health, Lahore, Pakistan

8 Department of Orthopedics, Tehsil Head Quarter, Ferozwala, Shaikhupura, Pakistan
9 Department of Biochemistry, University of Veterinary and Animal Sciences, Lahore, Pakistan

10 Medico Cirujano, ESACHS (Empresa de Servico Externo de la Asociacion Chilena de Seguridad), Punta Arenas, Chile

11 Department of Pharmacology and Toxicology, University of Veterinary and Animal Sciences, Lahore, Pakistan

12 Department of Nutrition, University of Veterinary and Animal Sciences, Lahore, Pakistan

13 Department of Community Medicine, Sahara Medical College, Narowal, Pakistan

14 Department of Internal Medicine, Mayo Hospital, Lahore, Pakistan

15 Department of Pediatrics, Sunny Downstate/Kings Country Medical Center, New York, NY, USA

16 Department of Community Medicine, Shaikh Khalifa Bin Zayed Al-Nahyan Medical and Dental College, Lahore, Pakistan 